\title{
Red blood cell creatine level to assess the need for and the therapeutic effect of red blood cell transfusions in hypovolemic and anemic patients to restore the red blood cell volume and RBC creatine to normal
}

\author{
CR Valeri*, GR Giorgio and CA Valeri \\ NBRL, Inc., Boston, MA, USA
}

\section{Introduction}

In 1969, the Naval Blood Research Laboratory (NBRL) at the Chelsea Naval Hospital, Chelsea, MA reported the increase in red blood cell 2,3 diphosphoglycerate (DPG) and red blood cell creatine levels in patients with red cell mass deficits or cardiopulmonary insufficiency [1]. The data indicated that the red blood cell 2,3 DPG and creatine levels assessed the state of tissue oxygenation and suggested that these two measurements could be used to evaluate the treatment of patients with red cell mass deficits and patients with cardiopulmonary insufficiency.

The red cell creatine levels were significantly correlated to the red cell mass deficits $(\mathrm{r}=0.52, \mathrm{p}<0.001)$ in 124 patients with various diagnoses. The correlation between red cell 2,3 DPG level and the red cell mass deficit was also significant $(\mathrm{r}=0.55, \mathrm{p}<0.001)$ in 123 patients. Likewise, there was a significant correlation between red cell creatine and 2,3 diphosphoglycerate concentration in 161 patients $(\mathrm{r}=0.60, \mathrm{p}<0.001)$.

Whole blood lactic acid concentrations varied from 0.5 to 3.0 $\mu$ moles per $\mathrm{mL}$ in 123 patients with varying degrees of red cell mass deficit. However, the correlation was not significant $(r=0.12, p>0.05)$ in 123 patients. The highest lactic acid concentrations were observed in the 28 patients with carcinoma.

Since 2,3 DPG has been shown to facilitate the release of oxygen from hemoglobin, we interpret our data to suggest that the increased 2,3 DPG concentration in the erythrocyte is a compensatory mechanism for delivering more oxygen to tissue. Although the mechanisms for the observed increases in red cell creatine and DPG are not known, the clinical importance of these red cell compounds deserves exploration because of their possible application both as diagnostic aids and for measuring the effectiveness of therapy. We have already shown that the measurement of red cell levels of 2,3 DPG in patients without cardiopulmonary disease is useful in assessing the indications for and effectiveness of red blood cell transfusions. Red cell 2,3 DPG measurements in patients with cardiopulmonary disease may help in the evaluation of the treatment of patients suffering from chronic pulmonary disease and congestive heart failure.

The measurement of red cell 2,3 DPG is affected by changes of acid-base balance, because acidosis decreases the red cell 2,3 DPG level whereas alkalosis increases this level. Since the measurement of 2,3 DPG is technically difficult and because of the excellent correlation between red cell levels of 2,3 DPG and creatine, the red cell creatine

measurement represents a simpler clinical assay for evaluating tissue hypoxia as a manifestation of both cardiopulmonary insufficiency and decreased circulating red cell mass.

Dr. Ernest Beutler in a commentary on the measurement of red cell creatine suggested that red cell creatine is metabolic garbage and has no known function $[2,3]$. In our study, patients with a decrease in red cell mass and with cardiopulmonary insufficiency produced red cells with increased 2,3 DPG and creatine levels. The release of muscle creatine occurred from the hypoperfused hypoxic muscles in these patients. Two red cell indicators of tissue hypoxia: increases in RBC 2,3 DPG and RBC creatine levels were observed in patients hospitalized at the Chelsea Naval Hospital [1]. Studies performed at the NBRL were unable to demonstrate that creatine could enter the RBC under the various in vitro conditions used in our investigations. However, in vivo studies showed that the increased RBC creatine levels observed in patients with chronic hypovolemic anemia of trauma following RBC transfusions to increase the peripheral red blood cell volume to normal were associated with a decrease in the red blood cell creatine level. In vivo studies suggested that red blood cell creatine level in these patients with chronic hypovolemic anemia of trauma correlated to the hypoxia in their muscles. The decrease in the peripheral red blood cell volume reduced the perfusion of the muscle of the extremities and increased the RBC DPG and creatine levels. Restoration of the peripheral blood volume by the red blood cell transfusions restored perfusion to the hypoxic muscles and the reduction in red blood cell DPG and creatine levels to normal with no change in the hematocrit [4,5].

The book The Hypovolemic Anemia of Trauma: The Missing Blood Syndrome published by CRC Press, Boca Raton, FL in 1981 was written to report on our clinical experience during the years 1968 to 1974 when over 300 patients who sustained war injuries in South Vietnam were transferred to Chelsea Naval Hospital, Chelsea, MA. The patients were called to the attention of our research facility when the use of general anesthesia for routine debridement of wounds precipitated a life-threatening state of hypotension. A series of tests established the presence of chronic hypovolemia [4,5].

*Correspondence to: CR Valeri, Naval Blood Research Laboratory, Inc., 195 Bournehurst Drive, Plymouth, MA 02360, USA, Tel. (508) 747-4472; E-mail: navblood@yahoo.com

Received: April 28, 2019; Accepted: May 23, 2019; Published: May 27, 2019 
Valeri CR (2019) Red blood cell creatine level to assess the need for and the therapeutic effect of red blood cell transfusions in hypovolemic and anemic patients to restore the red blood cell volume and $\mathrm{RBC}$ creatine to normal

When red blood cells were administered to the patients with this clinical disorder before their surgical procedure, hypotension during operations and post-operatively was eliminated. Determinations of blood volumes were made using $51 \mathrm{Cr}$ labeled autologous red blood cells to measure red blood cell volume and ${ }^{125} \mathrm{I}$ iodinated cold agglutinin to measure the plasma volume. Cardiac output and the distribution of blood flow were measured in these patients to understand how the vital organs such as the brain, heart, and kidneys were protected in spite of $30-40 \%$ reduction in total blood volume with normal or slightly reduced hematocrit.

To gain some understanding of the mechanism of the reduced blood volume, we studied the patient's red blood cell production as well as the destruction of the patient's own red blood cells and the donor red blood cells. Red blood cell production was impaired and both the patient and the donor RBC were being destroyed through an alternative pathway producing porphyrin-like substances instead of the usual urobilinogen breakdown product of the hemoglobin with the release of carbon monoxide. The degradation of red blood cell hemoglobin through the alternative pathway was thought to be responsible for the so-called missing blood syndrome [5].

The experience of Stinner and associates [6] is very different from our experience treating patients at the Chelsea Naval Hospital during the Vietnam War. They report that of 348 service members who sustained combat injuries in Afghanistan and Iraq, fifty-three (15.2\%) required amputations 12 weeks to 5.5 years following their initial extremity injuries [6].

In our patients at the Chelsea Naval Hospital although these orthopedic patients had chronic hypovolemic anemia of trauma with reduction in both the $\mathrm{RBC}$ and plasma volume, normal hemoglobin and hematocrit values, and normal central blood volumes, they suffered from severely contracted peripheral red blood cell volumes to their extremities. The aggressive transfusion of washed liquid preserved $\mathrm{RBC}$ and washed previously frozen deglycerolized RBC restored their peripheral red blood cell volume, the total blood volume, and the RBC 2,3 DPG and RBC creatine levels to normal with no change in the hematocrits $[4,5]$. We studied 300 servicemen for at least 2 years during their hospitalization at Chelsea Naval Hospital. Chronic hypovolemic patients with traumatic injuries to their extremities were transfused $\mathrm{RBC}$ in order to restore their peripheral red blood cell volume and to repair the injured extremities.

The recent paper by Stinner DJ and associates in Military Medicine reported the incidence of late amputation of $15 \%$ occurred 12 weeks to 5.5 years following combat related injuries sustained by U.S. military personnel in Afghanistan and Iraq [6]. During the Vietnam War from 1968 to 1974 our laboratory studied and treated over 300 wounded servicemen who returned to the Chelsea Naval Hospital from South Vietnam with severe traumatic injuries to their extremities who were treated by the orthopedic service. These patients with traumatic injuries to their extremities had chronic hypovolemic anemia of trauma with reduction in total blood volume of 30 to $40 \%$, normal hemoglobin and hematocrit values, normal central blood volume but severely reduced peripheral blood volume to their extremities. Aggressive transfusion of washed liquid preserved red blood cells and previously frozen deglycerolized red blood cells were administered to restore the peripheral blood volume to their extremities. The repeated red blood cell transfusion treatment to restore to normal the peripheral blood volume to their extremities was associated with repair of the wounded extremities with the rare need for an amputation in the 300 servicemen who were studied for one to two years during their hospitalization at the Chelsea Naval Hospital. Chronic hypovolemic patients with traumatic injuries to their extremities need to be transfused red blood cells to restore the peripheral blood volume to repair the injured extremities of these patients. Our studies demonstrate the limitation of the hematocrit and hemoglobin concentration measurements; the maintenance of the central blood volume and the $30-40 \%$ reduction in the peripheral blood volume in patients with chronic hypovolemia with traumatic injuries to their extremities. The repeated transfusions of viable and identifiable compatible red blood cells restored the peripheral red blood cell volume and permitted the healing of their injured extremities without the need for amputations. In these hypovolemic anemic patients both the RBC 2,3 DPG and RBC creatine levels were increased due to the muscle hypoxia associated with the reduction in the red blood cell volume. Red blood cell transfusions restored the RBC volume and reduced the increased levels of RBC 2,3 DPG and creatine to normal levels with no change in the hematocrit in these patients with traumatic injuries.

In the study of 300 wounded servicemen from Vietnam who were transferred to Chelsea Naval Hospital from 1968 to 1974, amputation of their extremities rarely occurred. The transfusion of these patients with washed liquid preserved red blood cells and washed previously frozen deglycerolized RBC increased the red blood cell volume and the plasma volume to their normal values and reduced the RBC 2,3 DPG and $\mathrm{RBC}$ creatine levels to normal with no change in the hematocrit. The multiple fractures of the upper and lower extremities healed without the need to amputate these extremities because of poor perfusion.

Studies in wounded servicemen that returned from Vietnam between 1968 to 1974 provided data to document the maintenance of the central blood volume with the reduction in the peripheral blood volume in hypovolemic anemic patients who were subjected to traumatic wounds. Transfusion of compatible but identifiable allogeneic red blood cells increased the blood volume to the gastrointestinal tract and muscle, bone and skin of the extremities. The increase in peripheral blood volume occurred following the transfusion of compatible and identifiable red blood cells. The studies performed in the chronic hypovolemic anemic patients demonstrated that transfusion of washed RBC not only increased the peripheral red blood cell volume but also increased the plasma volume. The red blood cell volume, the plasma volume and total blood volume in these chronic hypovolemic anemic patients were reduced. The normal hematocrit and hemoglobin concentration could not be used to assess the hypovolemic state in these patients with "stress anemia" [4]. The red blood cell volume was measured with 51Cr labeled autologous RBC and the plasma volume was measured with radiolabeled ${ }^{125}$ I cold agglutinin $19 \mathrm{~S}$ macroglobulin with a molecular weight of $1,000,000$. Radiolabeled human albumin could not be used to measure the plasma volume in patients because of the rapid loss of the radiolabeled albumin with molecular weight of 68,000 into the extravascular volume which overestimates the plasma volume [7]. The normal hematocrit in these patients reduced the nonsurgical blood loss from open wounds of the extremities which required surgical debridement.8 The chronic hypovolemic anemic patients subjected to general anesthesia for the debridement of their wounds of the extremities became hypotensive and a few of these wounded servicemen had cardiac arrests associated with the administration of general anesthesia. This observation confirmed that general anesthesia permitted redistribution of the blood volume from the central blood volume in the brain, heart, lungs, and kidneys into the hypoperfused peripheral blood volume which consists of the blood volume in the gastrointestinal tract, muscle, bones and skin of the extremities. In 
Valeri CR (2019) Red blood cell creatine level to assess the need for and the therapeutic effect of red blood cell transfusions in hypovolemic and anemic patients to restore the red blood cell volume and $\mathrm{RBC}$ creatine to normal

these patients with normal hematocrit and hemoglobin concentration values the debridement of wounds of the extremities was associated with minimal non-surgical blood loss from the open wounds of the extremities [8]. Another clinical observation was that following the transfusion of RBC which increased the peripheral red blood cell volume to the extremities, the edema in the tissues of the extremities disappeared. The reduction in the tissue edema in the extremities was associated with an increase in the plasma volume [9].

The transfusion of washed liquid preserved red blood cells and washed previously frozen deglycerolized red blood cells to chronic hypovolemic anemic patients increased both the red blood cell volume and the plasma volume. At the same time studies were done to assess whether washed autologous dog red blood cell transfusions would increase the plasma volume in dogs following acute hypovolemic anemia produced by removal of whole blood from the dogs. Dogs subjected to acute hypovolemia with reduced $\mathrm{RBC}$ volume and plasma volume were transfused with washed autologous dog red blood cells. Following transfusion, an increase in red blood cell volume and plasma volume occurred. The study demonstrated that plasma volume increased following the transfusion of washed autologous dog red blood cells to acute hypovolemic dogs. These studies indicated that the red blood cell volume regulated the plasma volume and the total blood volume in chronic hypovolemic patients and in acute hypovolemic dogs [9].

The hematocrit and the hemoglobin concentration of the chronic hypovolemic anemic patients did not increase following the transfusion of several units of compatible identifiable viable RBC because the plasma volume of the patients increased [9]. Both the red blood cell 2,3 DPG and the RBC creatine levels decreased to normal levelsfollowing the restoration of the red blood cell volume in these patients. The chronic hypovolemic anemic patients had normal hematocrit values and hemoglobin concentrations, but they had significantly elevated RBC 2,3 DPG levels and RBC creatine levels prior to the RBC transfusions. The aggressive transfusions of the chronic hypovolemic anemic patients with extremity injuries to restore the peripheral blood volume prevented the need to amputate extremities in the 300 wounded servicemen hospitalized at the Chelsea Naval Hospital between 1968 to 1974 [5].

The increase in RBC 2,3 DPG level was a compensatory mechanism to permit the reduced volume of red blood cells to improve oxygen delivery to tissue to maintain high $\mathrm{p}_{2}$ tensions in the brain, heart and kidneys without the need to increase blood flow to the brain, heart, and kidneys. The hypoperfusion of the muscle permitted leakage of creatine from the muscle which accumulated in the circulating RBC to increase the RBC creatine level. In patients with cardiopulmonary insufficiency increases in RBC 2,3 DPG and creatine levels were observed. Again, the tissue hypoxia produced in patients with cardiopulmonary insufficiency stimulated the RBC to increase the 2,3 DPG level to improve oxygen release from the RBC. The tissue hypoxia that was present in patients with cardiopulmonary insufficiency permitted loss of creatine from the hypoxic muscle which accumulated in the red blood cells. Both the red blood cell creatine and 2,3 DPG levels were elevated in patients with cardiopulmonary insufficiency reflected tissue hypoxia in the non-vital tissues like the muscle of the extremities.

The aggressive transfusion of the patients with traumatic injuries who had multiple fractures of the upper and lower extremities restored the blood volume to these areas. In the study of 300 wounded servicemen from Vietnam who were transferred to Chelsea Naval
Hospital from 1968 to 1974 , amputation of their extremities rarely occurred. The transfusion of these patients to restore the red blood cell volume, especially the peripheral red blood cell volume to extremities with washed liquid preserved red blood cells and washed previously frozen deglycerolized RBC increased the red blood cell volume, the plasma volume and total blood volume towards their normal values. The multiple fractures of the upper and lower extremities healed without the need to amputate these extremities because of poor perfusion.

The effect of the transfusion of washed red blood cells to restore the red blood cell volume and the plasma volume was associated with improvement in blood flow to the gastrointestinal tract and increase in appetite, improvement in libido, improvement in general health and decrease in the edema which was present in extremity wounds. The increase in red blood cell volume restored perfusion to the extremities with only rare amputation of the extremities by the aggressive transfusion to increase the peripheral red blood cell volume. The increase in the plasma volume associated with the transfusion of washed red blood cells was associated with decrease in the edema in the wounds of the extremities. Restoration of RBC volume to normal by the red blood cell transfusions was associated with reduction to normal of the RBC 2,3 DPG and RBC creatine levels with no change in the hematocrit $[5,9]$.

The therapeutic effectiveness of the transfusion of compatible, identifiable, viable washed red blood cells produced a significant reduction in both the elevated RBC 2,3 DPG and creatine levels. The elevated red blood cell creatine level could not be explained by an increased in red blood cell production which was reduced in these patients. The increased red blood cell creatine in these patients with chronic hypovolemia of trauma was not due to an increase in erythropoiesis. RBC creatine level correlated to the red blood cell volume deficit and not to the mean red blood cell age [5].

The study of the patients wounded in Vietnam revealed that their wounds required debridement under anesthesia, but bleeding of these wounds was minimal. The reduction in nonsurgical blood loss observed with debridement of these wounds of the extremities was related to the normal or slightly reduced hematocrit of these patients. This serendipitous clinical observation stimulated the NBRL to study the effect of hematocrit on the bleeding time and nonsurgical blood loss. Viable and functional RBC reduce the bleeding time and nonsurgical blood [8].

The paper by Stinner DJ and associates in Military Medicine reported the incidence of late amputation of $15 \%$ occurred 12 weeks to 5.5 years following combat related injuries sustained by U.S. military personnel in Afghanistan and Iraq [6]. During the Vietnam War from 1968 to 1974 our laboratory studied and treated over 300 wounded servicemen who returned to the Chelsea Naval Hospital from South Vietnam with severe traumatic injuries to their extremities who were treated by the orthopedic service. These patients with traumatic injuries to their extremities had chronic hypovolemic anemia of trauma with reduction in total blood volume of 30 to $40 \%$, normal hemoglobin and hematocrit values, normal central blood volume but severely reduced peripheral blood volume to their extremities and increased RBC 2,3 DPG and RBC creatine levels. Aggressive transfusion of washed liquid preserved red blood cells and previously frozen deglycerolized red blood cells were administered to restore the peripheral blood volume to their extremities. The repeated red blood cell transfusion treatment to restore to normal the peripheral blood volume to their extremities was associated with repair of the wounded extremities with the rare 
Valeri CR (2019) Red blood cell creatine level to assess the need for and the therapeutic effect of red blood cell transfusions in hypovolemic and anemic patients to restore the red blood cell volume and $\mathrm{RBC}$ creatine to normal

need for an amputation in the 300 servicemen who were studied for one to two years during their hospitalization at the Chelsea Naval Hospital. The book the Hypovolemic Anemia of Trauma: The Missing Blood Syndrome by Valeri CR and Altschule MD, CRC Press, Boca Raton, FL, 1981, was written to report that chronic hypovolemic patients with traumatic injuries to their extremities need to be transfused red blood cells to restore the peripheral blood volume to repair the injured extremities of these patients. The studies reported in the book demonstrate the limitation of the hematocrit and hemoglobin concentration measurements; the maintenance of the central blood volume and the $30-40 \%$ reduction in the peripheral blood volume in patients with traumatic injuries to their extremities with increased RBC 2,3 DPG and RBC creatine levels. The repeated transfusions of viable and identifiable compatible red blood cells restored the peripheral blood volume and permitted the healing of their injured extremities without the need for amputations.

Red blood cell transfusions are indicated for patients with hypovolemic anemia of trauma with normal hematocrit and hemoglobin levels to restore the peripheral red blood cell volume to normal to prevent the need for amputations. The red blood cell creatine level is not metabolic garbage but a measurement of muscle hypoxia. An increase in red blood cell creatine measures the reduced oxygen tension in muscle and red blood cell transfusions are indicated to restore the peripheral red blood cell volume to normal and to reduce the red blood cell creatine to normal in hypovolemic patients with traumatic injuries to prevent the need for amputations.

Studies from the NBRL, Boston, MA have reported that the patient's peripheral venous hematocrit $(\mathrm{Hct})$ should not be the sole determinant of the red blood cell (RBC) transfusion trigger. The Hct reflects the concentration of the RBCs in the blood not the RBC volume, plasma volume, or total blood volume nor does the hematocrit indicate whether the patient is hypovolemic, normovolemic, or hypervolemic. Studies in more than 300 wounded servicemen who were injured in South Vietnam and transferred to the Chelsea Naval Hospital, Chelsea, MA had reductions of 30 to 40 percent in the red blood cell volume, plasma volume, and the total blood volume. The red blood cell volume was measured with $51 \mathrm{Cr}$ labeled autologous RBCs and the plasma volume was measured with ${ }^{125} \mathrm{I}$ labeled cold agglutinin. These hypovolemic anemic patients had normal peripheral venous Hct and hemoglobin concentrations $[4,5]$. The limitation of the hematocrit to assess the need for red blood cell transfusion in hypovolemic anemic patients was reported by Valeri CR and associates [10].

In this study the patients' RBC volumes were measured with $51 \mathrm{Cr}$ and $99 \mathrm{mTc}$-radiolabeled fresh autologous RBCs. The total blood volume and the plasma volume were estimated from the radioisotope labeled RBC volume and the total body Hct (peripheral venous hematocrit multiplied by 0.89 ). The measured RBC volume, plasma volume, and total blood volume for each patient were compared to preoperative values.

Two patient populations were studied: 41 consecutive patients subjected to elective vascular surgery and 20 consecutive patients subjected to cardiopulmonary bypass surgery. The RBC volume was measured with $51 \mathrm{Cr}$ or $99 \mathrm{mTc}$-labeled autologous fresh RBCs, and the plasma volume and total blood volume were estimated from the measured RBC volume and the total body Hct level (peripheral venous hematocrit multiplied by 0.89). Measurements made 1 to 2 and 24 hours after surgery were compared to the preoperative values for these two groups of patients.
During the 24-hour posttransfusion period, the RBC, plasma, and total blood volumes were reduced compared to the preoperative volumes. These patients were hypovolemic and anemic and their Hct values during the 24 -hour postoperative period were increased by a mean of 4 to 5 volume percent compared to values that would be expected if they were normovolemic and anemic. The Hct values in hypovolemic anemic patients are elevated because the plasma volume does not increase to achieve the normovolemic anemic state.

In the 41 patients subjected to the vascular surgical procedures, the Hct values 1 to 2 and 24 hours after the surgical procedure correlated significantly to the $\mathrm{RBC}$ volume $(\mathrm{p}<0.05$ to $\mathrm{p}<0.001)$ and the $\mathrm{RBC}$ deficit $(\mathrm{p}<0.01$ to $\mathrm{p}<0.001)$ but not to the total blood volume $(\mathrm{p}>0.05)$.

In the 20 patients subjected to cardiopulmonary bypass surgical procedures, the Hct values 1 to 2 and 24 hours after the surgical procedure correlated significantly to the $R B C$ volume $(\mathrm{p}<0.05$ to $\mathrm{p}<0.001)$ and the $R B C$ volume deficit $(\mathrm{p}<0.05$ to $\mathrm{p}<0.001)$ but not to the total blood volume $(\mathrm{p}>0.05)$. For all the patients the Hct values 1 to 2 and 24 hours after the surgical procedure correlated significantly with the $R B C$ volume $(\mathrm{p}<0.001)$ and the $R B C$ volume deficits $(\mathrm{p}<0.001)$ but the Hct did not correlate with the total blood volume ( $\mathrm{p}>0.05)$.

The patients subjected to vascular surgical procedures were hypovolemic and anemic and had significantly reduced RBC volumes and significantly reduced total blood volumes 1 to 2 hours $(\mathrm{p}<0.01$, $\mathrm{p}<0.001)$ and 24 hours postoperatively $(\mathrm{p}<0.001, \mathrm{p}<0.05)$ compared to their preoperative measured RBC and total blood volumes. The mean Hct level was 38 percent 1 to 2 hours postoperatively and 35 percent 24 hours postoperatively. If these patients had been normovolemic with reduced RBC volume, their mean Hct value would have been 32 volume-percent (Figure 1). Cardiac output in these patients was significantly increased 1 to $2(\mathrm{p}<0.05)$ and 24 hours postoperatively $(\mathrm{p}<0.01)$ compared to the preoperative output. The mean arterial pressure was significantly reduced 1 to $2(\mathrm{p}<0.001)$ and 24 hours postoperatively $(\mathrm{p}<0.001)$ and the mean heart rate was significantly increased 1 to $2(\mathrm{p}<0.001)$ and 24 hours postoperatively $(\mathrm{p}<0.001)$ compared to the preoperative values.

The 20 patients subjected to cardiopulmonary bypass surgical procedures were hypovolemic and anemic with significantly reduced RBC volumes and significantly reduced total blood volumes 1 to 2 $(\mathrm{p}<0.001, \mathrm{p}<0.01)$ and 24 hours postoperatively $(\mathrm{p}<0.001, \mathrm{p}<0.01)$ compared to the preoperative measured $\mathrm{RBC}$ and total blood volume. The mean Hct value was 30 volumes percent 1 to 2 hours postoperatively and 27 volumes percent 24 hours postoperatively, if these patients had been normovolemic with reduced RBC volumes, their mean Hct values would have been 25 and 23 volumes percent during these postoperative periods (Figure 2). The cardiac output in these patients 1 to 2 ( $\mathrm{p}=\mathrm{NS}$ ) and 24 hours postoperatively ( $\mathrm{p}=\mathrm{NS}$ ) was not significantly different from the preoperative output. The mean arterial pressure was significantly reduced 1 to $2(\mathrm{p}<0.01)$ and 24 hours postoperatively $(\mathrm{p}<0.05)$ and the mean heart rate was significantly increased 1 to $2(\mathrm{p}<0.01)$ and 24 hours postoperatively $(\mathrm{p}<0.05)$ compared to the preoperative values.

In all 61 patients in this study the Hct values correlated with the $\mathrm{RBC}$ volumes and with the RBC deficits but did not correlate with the total blood volume. All 61 patients were hypovolemic with significantly reduced $\mathrm{RBC}$ volume during the 24 -hour postoperative period.

Anemia is defined as a reduction in $\mathrm{RBC}$ volume and the $\mathrm{RBC}$ volume deficit is assessed clinically from the peripheral venous $\mathrm{Hct}$ value. The purpose of our study was to assess the Hct level, the RBC 


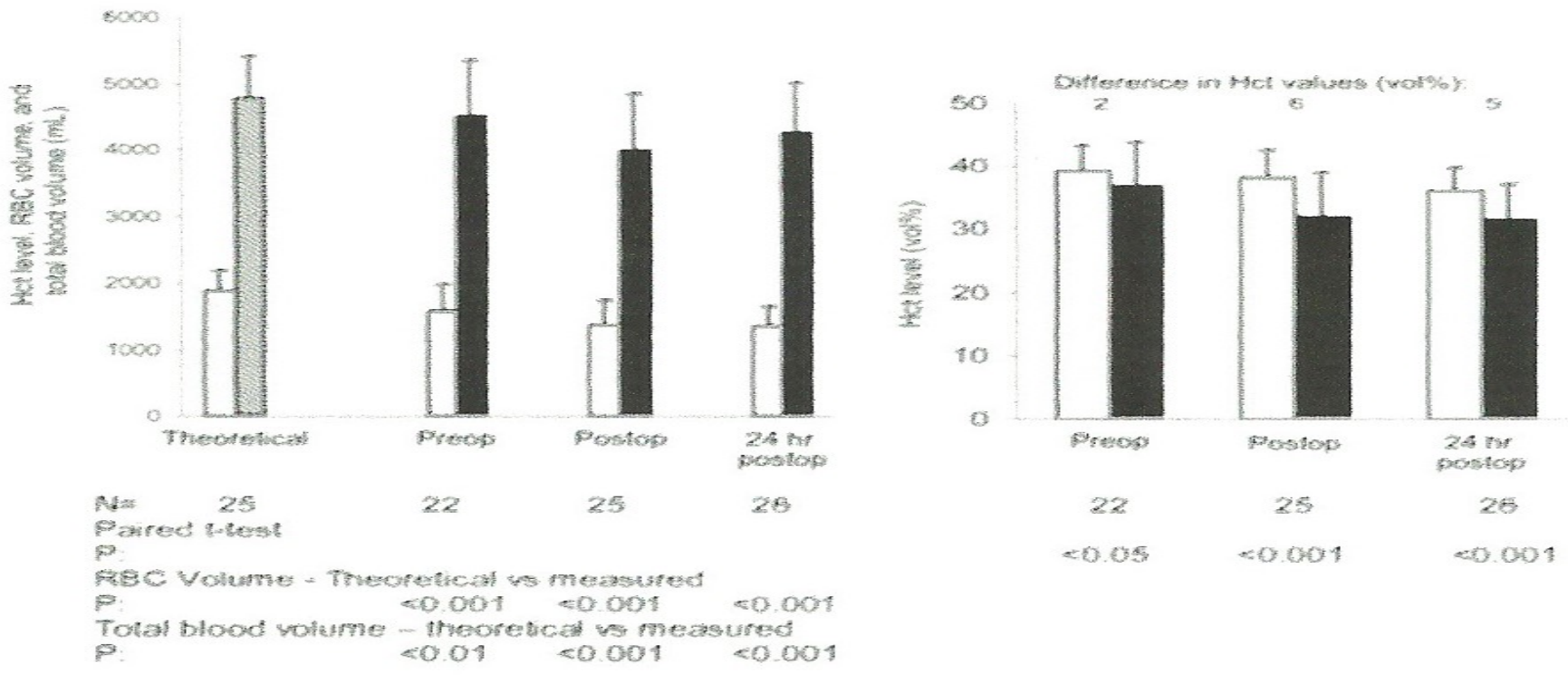

Figure 1. (Left) Peripheral venous Hct level, theoretical RBC volume ( $\square$ ), and total blood volume before surgery (Preop) and at 1 to 2 and 24 hours postoperatively (Postop) in patients subjected to vascular surgery. (Right) The measured peripheral venous Hct level in the patients who were hypovolemic and anemic $(\square)$ and the expected peripheral venous Hct if the patients were normovolemic and anemic $(\square)$ are reported (mean \pm SD) [10]

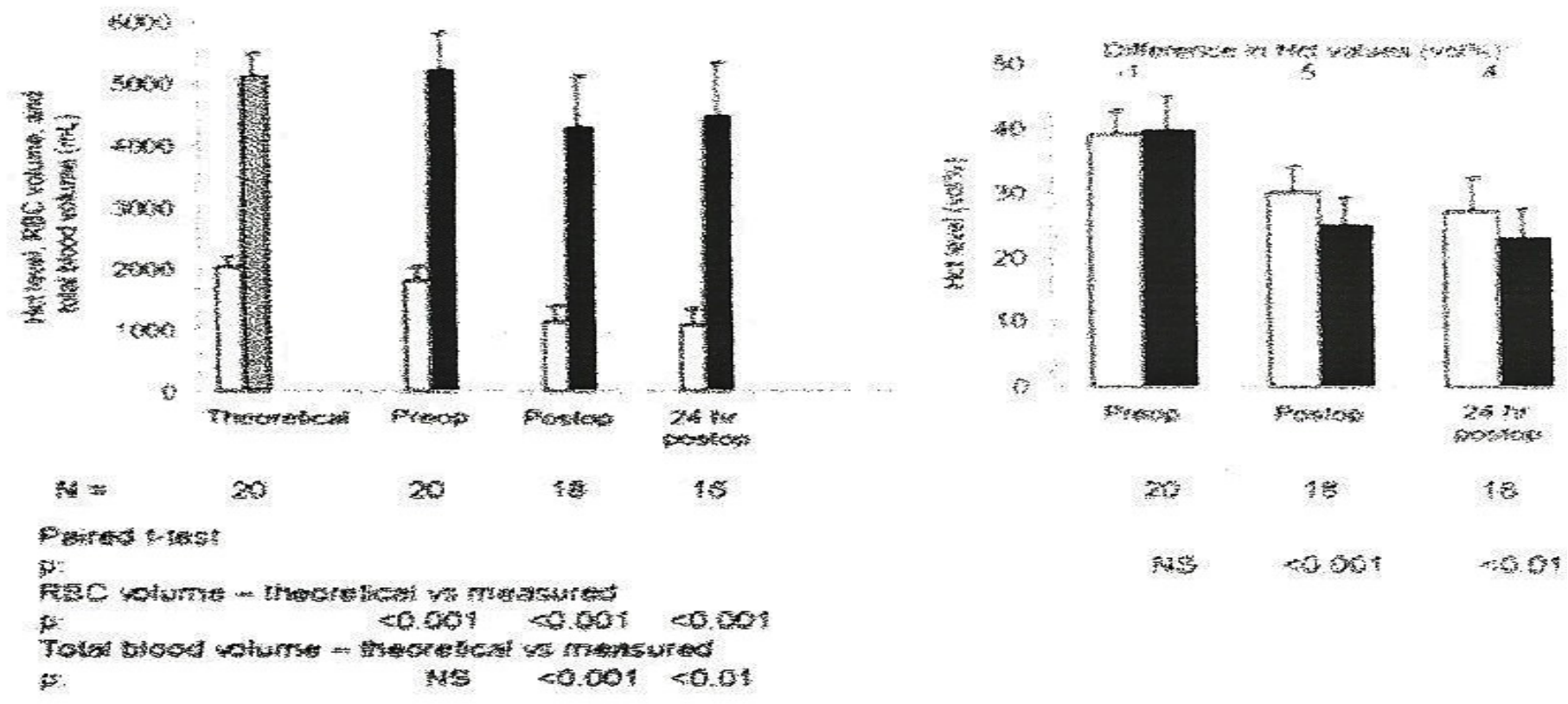

Figure 2. (Left) Peripheral venous Hct level, theoretical RBC volume ( $\square$ ), and total blood volume ( $\square$ ) before surgery and the $99 \mathrm{mTc}$ RBC volume ( $\square$ ) and total blood volume ( before surgery (Preop) and at 1 to 2 and 24 hours postoperatively (Postop) in patients subjected to cardiopulmonary surgery. (Right) The measured peripheral venous Hct level in the patients who were hypovolemic and anemic $(\square)$ and the expected peripheral venous Hct if the patients were normovolemic and anemic ( $\square$ ) are reported (mean \pm SD) [10]

volume, the plasma volume, and the total blood volume before and during the 24-hour postoperative period in patients subjected to vascular and cardiopulmonary bypass surgical procedures. The RBC volumes were measured with 51Cr labeled autologous fresh RBCs in the patients subjected to vascular surgical procedures and with $99 \mathrm{mTc}$ labeled fresh autologous RBC in the patients subjected to the cardiopulmonary bypass procedure. The $51 \mathrm{Cr}$ and $99 \mathrm{mTc}$ labeling procedures have been shown to provide similar RBC volumes in patients and baboons. Jones and Mollison [11] have reported no differences in RBC volumes in patients whether measurements were made with $51 \mathrm{Cr}$ or $99 \mathrm{mTc}$ labeled fresh autologous RBCs, and our data from a baboon study confirmed this finding [12].

When ${ }^{125} \mathrm{I}$ labeled albumin is used to measured plasma volume in patients, the plasma volume is overestimated because the ${ }^{125} \mathrm{I}$ labeled albumin with a molecular weight of 68,000 is distributed rapidly into 
Valeri CR (2019) Red blood cell creatine level to assess the need for and the therapeutic effect of red blood cell transfusions in hypovolemic and anemic patients to restore the red blood cell volume and $\mathrm{RBC}$ creatine to normal

the extravascular space [5,7] Previous studies in our laboratory have albumin does [5,7]. state (Figure 3). regarding the need for $\mathrm{RBC}$ transfusions. shown that accurate plasma volume measurements can be made in patients with ${ }^{131} \mathrm{I}$ labeled cold agglutinin because this $19 \mathrm{~S}$ molecule does not exit the intravascular volume during the mixing time as ${ }^{125} \mathrm{I}$ labeled

The estimates of the theoretical RBC volume, plasma volume, and total blood volume were made from coefficients provided by M. Strumia, Chairman of the National Research Council, in 1972. Our laboratory reported coefficients for the theoretical RBC volume, plasma volume, and total blood volume for health male volunteers in a collaborative study with the US Army Research Institute of Environmental Medicine (Natick, MA) in 1992, which are similar to those provided by M. Strumia and used in this study [10,13].

The measured RBC and total blood volume 1 to 2 and 24 hours after surgery were compared to the measured RBCs and total blood volumes before surgery. In the two groups of patients, the measured RBC volume and total blood volumes 1 to 2 and 24 hours after the surgery were significantly $(\mathrm{p}<0.05)$ reduced compared to the preoperative values. In the two groups, the hypovolemic anemic state was associated with an elevated Hct level of a mean of 4 to 5 volume percent (Figure 1 and 2) because the plasma volume did not increase to compensate for the reduction in the $\mathrm{RBC}$ volume to produce the normovolemic anemic

Data from the clinical study reported in Ref 10 demonstrated that all the patients had acute hypovolemic anemia with significantly reduced $51 \mathrm{Cr}$ and $99 \mathrm{mTc} \mathrm{RBC}$ volumes and significantly reduced total blood volumes during the 24 -hour postoperative period compared to the measured preoperative values. The postoperative Hct values were lower in patients after cardiopulmonary bypass surgery procedures than after the vascular surgical procedure owing to the fact that hemodilution was performed in the cardiopulmonary bypass patients. In all the patients, the Hct values correlated with the RBC volume and with the RBC deficit during the postoperative periods but did not correlate with the total blood volumes. The significant correlations between the Hct and the $\mathrm{RBC}$ volume and the RBC deficit do not provide quantitative data

\section{Normovolemia}

With normal

Het

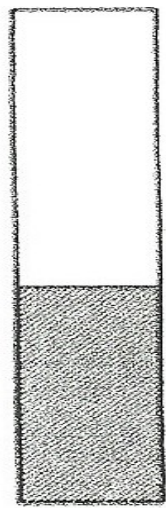

Het

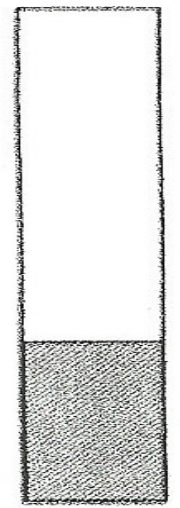

The use of the Hct value as the transfusion trigger assumes that the patient has normovolemic anemia. The Hct level does not differentiate between hypovolemic and normovolemic patients. The normovolemic state is usually determined from measurements of mean arterial pressure, cardiac output, heart rate, arterial blood gases $\left(\mathrm{pH}, \mathrm{pCO}_{2}\right.$, $\mathrm{pO}_{2}$ ) and renal function, all measurements that assess the central blood volume but not the total blood volume. Total blood volume includes both the central blood volume, which includes the blood volume in the heart, lungs, brain, and kidneys, and the peripheral blood volume, which includes the blood volume in the skin, extremities, and the gastrointestinal tract $[5,14]$.

In a patient with hypovolemic anemia, the Hct value is elevated because the plasma volume does not increase to achieve a state of normovolemic anemia. Hypovolemic patients with reduced RBC and plasma volumes may have normal or reduced Hct values and undetectable or underestimated anemia (Figure 3) [5,14]. We do not know why the plasma volume did not increase in these patients with acute hypovolemic anemia after vascular and cardiopulmonary bypass surgical procedures or in the previously studied wounded servicemen with chronic hypovolemic anemia. In our previous studies, we found that when we washed the liquid preserved $\mathrm{RBC}$ and previously frozen RBCs before transfusion to wounded servicemen with chronic hypovolemic anemia RBC volume, plasma volume, and total blood volume increased but there was no significant change in the Hct value after the transfusion of 3 to 4 units of washed RBCs $[5,14]$. In a previous study in which dogs were subjected to acute hypovolemic anemia, the transfusion of washed autologous dog RBCs increased both the RBC and the plasma volume [9]. These findings in wounded servicemen with chronic hypovolemic anemia and in dogs with acute hypovolemic anemia suggest that the $\mathrm{RBC}$ volume may regulate the plasma volume.

The Hct level is used as the major trigger for RBC transfusion even though it cannot identify the hypovolemic state in patients with normal or slightly reduced Hct values who have significant reductions in RBC volume, and this could result in hypovolemic anemic patients not being adequately transfused (Figure 3 ).

\section{Hypovolemia}

With increased With slightly

Het reduced Hct

With reduced

Hct
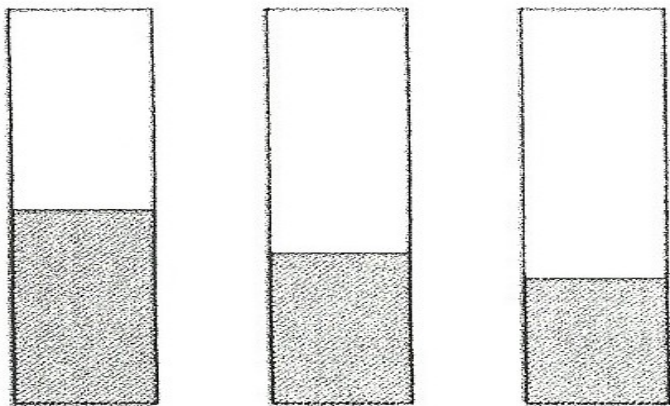

Het range

$(\operatorname{vol} \%)$

$42-45$

$30-35$

$50-55$

$38-40$

30-35

Figure 3. The peripheral venous Hct level, the RBC volume, plasma volume and total blood volume in normovolemic and hypovolemic patients [10] 
Valeri CR (2019) Red blood cell creatine level to assess the need for and the therapeutic effect of red blood cell transfusions in hypovolemic and anemic patients to restore the red blood cell volume and $\mathrm{RBC}$ creatine to normal

The limitation of the hematocrit to assess the need for red blood cell transfusions in hypovolemic anemic patients to restore the red blood cell volume to normal was documented by studies published by the NBRL, Boston, MA $[4,5,10,14]$. Three hundred (300) servicemen injured in Vietnam were transferred to the Chelsea Naval Hospital, Chelsea, MA for treatment between 1968 to 1974 [4,5,14]. These wounded servicemen with orthopedic injuries had chronic hypovolemic anemia of trauma with 30 to $40 \%$ reduction in the total blood volume measured by $51 \mathrm{Cr}$ labeled autologous red blood cell volume and ${ }^{125}$ I labeled cold agglutinin plasma volume, normal hematocrit and hemoglobin concentration and increased RBC 2,3 DPG and RBC creatine levels. The repeated transfusion of liquid preserved washed $\mathrm{RBC}$ and previously frozen deglycerolized RBC increased the $51 \mathrm{Cr}$ red blood cell volume to normal, restored the plasma volume to normal and the total blood volume to normal and reduced RBC 2,3 DPG and $\mathrm{RBC}$ creatine levels to normal [14].

In 1969 the NBRL reported that RBC 2,3 DPG and RBC creatine levels measured tissue hypoxia in patients with red blood cell mass deficits [1]. The increase RBC 2,3 DPG and RBC creatine levels indicated the need for RBC transfusion and the therapeutic effect of red blood cell transfusions to restore to normal the red blood cell volume of these hypovolemic anemic patients. The increases in RBC 2,3 DPG and $\mathrm{RBC}$ creatine levels occurred with reduction in the red blood cell volume. Restoration of the RBC volume by transfusion of compatible and viable $\mathrm{RBC}$ reduced the RBC 2,3 DPG and RBC creatine levels to normal $[4,5,14]$

In the study of hypovolemic anemic patients, the hematocrit and hemoglobin concentration could not be used to assess the need for red blood cell transfusions and the therapeutic effect of red blood cell transfusions to restore the red blood cell volume to normal $[5,10,14]$.

In hypovolemic anemic patients the decrease in the red blood cell volume was associated with an increase in both the RBC 2,3 DPG and $\mathrm{RBC}$ creatine levels. $\mathrm{RBC}$ creatine level is easier to measure than $\mathrm{RBC}$ 2,3 DPG level and is not affected by the blood $\mathrm{pH}$ because acidosis decreases the RBC 2,3 DPG level whereas alkalosis increases the RBC 2,3 DPG level.

The data reported in references 5 and 14 show that patients with hypovolemic anemia with normal hematocrit and hemoglobin concentration have elevated $\mathrm{RBC}$ creatine levels and with the restoration of the 51Cr RBC volume to normal by red blood cell transfusion of compatible and viable $\mathrm{RBC}$ the $\mathrm{RBC}$ creatine level decreased to its normal level. In hypovolemic anemic patients the elevated RBC creatine level and not the hematocrit and hemoglobin concentration should be measured to assess the need for and the therapeutic effect of RBC transfusions to restore to normal the red blood cell volume. The RBC creatine should be measured to assess both the need for and the therapeutic effect of compatible and viable red blood cell transfusions to restore the $\mathrm{RBC}$ volume to normal and reduce the red blood cell creatine to normal in patients with hypovolemic anemia. The transfusions of compatible and viable RBC are indicated to increase the red blood cell volume to normal, the plasma volume to normal, and the total blood volume to normal, and reduce the RBC creatine to normal in hypovolemic anemic patients.

\section{Acknowledgements}

To the memory of Domenica Piccolomini, Angelo Valeri, Louise Ragno, and Nicholas Ragno.

\section{References}

1. Valeri CR, Fortier NL (1969) Red-cell 2,3-diphosphoglycerate and creatine levels in patients with red-cell mass deficits or with cardiopulmonary insufficiency. NEJM 281: 1452-1455. [Crossref]

2. Beutler E (1970) Is red-cell creatine metabolic garbage? N Engl J Med 282: 979-980 [Crossref]

3. Valeri CR, Fortier NL (1970) Is red-cell creatine metabolic garbage? NEJM 282: 979980. [Crossref]

4. Biron PE, Howard J, Altschule MD, Valeri CR (1972) Chronic deficits in red-cell mas in patients with orthopaedic injuries (stress anemia). J Bone Joint Surg 54-A: 10011014.

5. Valeri CR and Altschule MD (1981) Hypovolemic Anemia of Trauma: The Missing Blood Syndrome. Chemical Rubber Company, Boca Raton, Florida.

6. Stinner DJ, Burns TC, Kirk KL, Scoville CR, Ficke JR, et al. (2010) Prevalence of late amputations during the current conflicts in Afghanistan and Iraq. Mil Med 175: 1027-1029. [Crossref]

7. Valeri CR, Cooper AG, Pivacek LE (1973) Limitations of measuring blood volume with iodinated $\mathrm{I}^{125}$ serum albumin. Arch Intern Med 132: 534-538. [Crossref]

8. Valeri CR, Cassidy G, Pivacek LE, Ragno G, Lieberthal W, et al. (2001) Anemiainduced increase in the bleeding time: indications for treatment of nonsurgical blood loss. Transfusion 41: 977-983. [Crossref]

9. Valeri CR, Donahue K, Feingold HM, Cassidy GP, Altschule MD (1986) Increase in plasma volume after the transfusion of washed erythrocytes. Surg Gynec Obstet 162: 30-36. [Crossref]

10. Valeri CR, Dennis RC, Ragno G, MacGregor H, Menzoian JO, et al. (2006) Limitations of the hematocrit to assess the need for RBC transfusion in hypovolemic anemic patients. Transfusion 46: 365-371. [Crossref]

11. Jones J, Mollison PL (1978) A simple and efficient method of labelling red cells with 99mTc for determination of red cell volume. Br J Haematol 38: 141-148. [Crossref]

12. Valeri CR, MacGregor H, Giorgio A, Srey R, Ragno G (2003) Comparison of radioisotope methods and a non-radioisotope method to measure the $\mathrm{RBC}$ volume and RBC survival in the baboon. Transfusion 43: 1365-1373. [Crossref]

13. Sawka MN, Young AJ, Pandolf KB, Dennis RC, Valeri CR (1992) Erythrocyte, plasma and blood volume of healthy young men. Med Sci Sports Exerc 24: 447-453. [Crossref]

14. Valeri CR, Giorgio GA, Valeri CA (2018) The indications for RBC transfusions to restore the peripheral red blood cell volume in patients with hypovolemic anemia of trauma to prevent amputations. Trauma Emerg Care 31: 1-10.

Copyright: (C2019 Valeri CR. This is an open-access article distributed under the terms of the Creative Commons Attribution License, which permits unrestricted use, distribution, and reproduction in any medium, provided the original author and source are credited. 